\title{
Lexis
}

Journal in English Lexicology

Book reviews | 2018

Blandine PENNEC \& Olivier SIMONIN, Les locutions de l'anglais, emplois et stratégies rhétoriques. Fixed Phrases in English, Use and Rhetorical Strategies.

Presses Universitaires de Perpignan, 2011, 240 pages.

\section{Frédérique Brisset}

\section{(2) OpenEdition}

Journals

Electronic version

URL: http://journals.openedition.org/lexis/2506

DOI: $10.4000 /$ lexis.2506

ISSN: 1951-6215

Publisher

Université Jean Moulin - Lyon 3

Electronic reference

Frédérique Brisset, «Blandine PENNEC \& Olivier sIMONIn, Les locutions de l'anglais, emplois et stratégies rhétoriques. Fixed Phrases in English, Use and Rhetorical Strategies. », Lexis [Online], Book reviews, Online since 06 December 2018, connection on 24 September 2020. URL : http://journals.openedition.org/ lexis/2506 ; DOl : https://doi.org/10.4000/lexis.2506

This text was automatically generated on 24 September 2020.

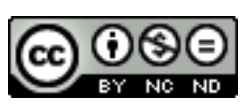

Lexis is licensed under a Creative Commons Attribution-NonCommercial-NoDerivatives 4.0 International License. 


\section{Blandine PENNEC \& Olivier SIMONIN, Les locutions de l'anglais, emplois et stratégies rhétoriques. Fixed Phrases in English, Use and Rhetorical Strategies.}

Presses Universitaires de Perpignan, 2011, 240 pages.

Frédérique Brisset

\section{REFERENCES}

Blandine Pennec \& Olivier Simonin

Les locutions de l'anglais, emplois et stratégies rhétoriques Fixed Phrases in English, Use and Rhetorical Strategies Presses Universitaires de Perpignan, Cahiers de l'Université de Perpignan, Perpignan, 2011. ISBN : 978-2-35412-145-7, Prix : 18€, 240 pages.

1 Quelles que soient les langues concernées, les locutions sont un phénomène polymorphe, dont la typologie ne fait pas l'unanimité. Quelques critères saillants apparaissent toutefois de façon récurrente ; on peut, a minima, en citer trois proposés par Mitterand [1976:61], qui renvoient aux notions de figement et d'idiomaticité : leur « intégrité morpho-syntaxique », leur « intégrité grammatico-sémantique ([...] qui ne peut être désintégrée sans que chacun des composants perde sa valeur figurée) » et la «fréquence remarquable de leur emploi pour désigner un concept unique ». Le mérite majeur de l'ouvrage bilingue présenté ici, évident dès son sommaire, est dès lors sa large acception de la notion de locution et la traduction même de celle-ci dans son titre (fixed phrases) en témoigne. En effet, même si la définition de ce concept phraséologique reste sujette à caution ${ }^{1}$, les auteurs des différents articles se plaisent tous à le remarquer, cet inconvénient est aussi synonyme d'une grande richesse et variété d'objets de recherches et, en conséquence, d'approches méthodologiques et scientifiques. 
2 La sélection de huit études proposées dans ce recueil suite au colloque éponyme tenu en 2010 à Perpignan (six en anglais et deux en français) s'intéresse à des domaines aussi divers que, notamment, l'apprentissage d'une langue seconde, les expressions basées sur des chiffres ou les tournures employées dans le monde footballistique, et convoque à la fois lexicologie et syntaxe, pragmatique et linguistique, rhétorique et stylistique. Cette diversité reflète l'omniprésence des locutions en langue anglaise et leur hétérogénéité, soulignées par les directeurs du volume dans leur introduction, offerte en deux versions, française et anglaise (p. 12).

3 Amanda Edmonds (p. 27) offre ainsi, dès le premier article, un projet ambitieux : « Setting boundaries within the second language formulaic language landscape ». Elle pointe, grâce à un large panorama fort bien documenté, l'absence de consensus sur la définition même de locution, visible à travers l'abondante terminologie rencontrée dans la littérature scientifique en anglais, et la difficulté subséquente à identifier le phénomène. Après ce constat de quasi impuissance (p.38), la nécessité d'une classification fonctionnelle s'avère donc d'autant plus prégnante sur le plan didactique qu'elle aura un impact sur les stratégies d'apprentissage à mettre en œuvre. Suivant que l'on souscrit à une vision fonctionnelle et pragmatique de ces expressions, qui dépendraient de conventions liées au contexte d'usage ou, au contraire, à une vision psycholinguistique, qui les envisage en tant que formules enregistrées dans la mémoire comme des éléments de quasi " prêt-à-parler " linguistique ${ }^{2}$, la perspective de recherche ou le projet pédagogique sera bien évidemment orienté tout à fait différemment.

4 Alice Violet enchaîne ensuite avec une étude de cas sur les combinaisons Préposition + nom au singulier, dans l'optique de déterminer la valeur sémantique de l'article zéro caractéristique de ces locutions syntaxiques, car ces idiomatismes sont marqués par un fort degré de figement et des contraintes lexicales et grammaticales. Elle conduit sa démonstration à partir du British National Corpus, sur six noms de lieu méronymiques précédé de at (base, bottom, core, heart, root, source), dont l'étude diachronique montre une évolution sensible, du sens propre purement concret à celui de marqueur adverbial rhétorique ; l'article zéro signale alors ce glissement vers le sens figuré, où la locution sert désormais au locuteur à souligner la pertinence de son discours.

5 Bertrand Richet (p. 111) a choisi, pour sa part, de se pencher sur les locutions faisant appel aux nombres, qui sont nombreuses en toutes langues. Son étude sur corpus s'attache surtout aux dimensions statistiques et reste malheureusement fort descriptive (est-il bien nécessaire de rappeler au lecteur, par exemple, que les unités sont les chiffres de 0 à 9 , les dizaines de 10 à 99, etc. ?). Sur ce sujet pourtant prometteur, il souligne d'ailleurs lui-même la difficulté à passer de l'analyse quantitative à l'analyse qualitative. En choisissant de se consacrer principalement à one, two et three, qui sont les plus représentés en locutions, il tente de montrer comment ces dernières, porteuses d'une valeur souvent symbolique, illustrent le passage de l'unité au système puis à la série.

6 Jim Walker s'intéresse, lui, aux clichés typiques du discours footballistique, dont il défend l'intérêt comme objet de recherche. Bien sûr, on connaît la fortune de ce vocabulaire quelque peu cryptique qui a souvent essaimé hors de ce technolecte, mais Walker, en adoptant une perspective diachronique, montre combien ce phénomène dépasse le seul plan lexicologique. Après avoir redéfini la notion de cliché, il retrace le cheminement de ces éléments de discours, en balayant des occurrences qui affectent 
également la phraséologie et la grammaire, avec dans ce dernier cas l'exemple typique de l'essor d'un present perfect narratif. Ces formes ont en effet une tendance avérée à abonder l'anglais courant, ce qui ouvre des pistes de recherche significatives pour les linguistes, au-delà du jargon des seuls amateurs de ballon rond.

7 Christelle Lacassain-Lagoin s'interroge dans l'article suivant sur les verbes de perception dans les périphrases du type Have $a V$. Son étude très complète sur les corpus britannique BNC et américain COCA met en regard le degré de figement de ces locutions et leurs valeurs rhétoriques et discursives, montrant à partir de nombreuses occurrences comment elles se différencient des formes verbales simples sur le plan sémantique : à la différence des combinaisons give $a V$ et take $a V$, elles impliquent en effet une focalisation sur le sujet grammatical du verbe, en tant qu'agent dont la perception est ainsi actualisée et incluse dans le champ d'expérience personnel Bourdieu [1982: 100] n'écrivait-il pas : «le dicton, le proverbe et toutes les formes d'expression stéréotypées ou rituelles sont des programmes de perception»? L'auteure, après avoir testé les critères socio-linguistique et syntaxique sur les trois compositions (have, give et take), met en avant de manière très convaincante l'importance des facteurs sémantiques, souvent sous-estimés dans ce type d'étude.

Graham Ranger offre ensuite une réflexion détaillée sur le marqueur de discours You see comme opérateur métalinguistique à l'oral ; si celui-ci a souvent été envisagé dans le cadre de recherches pragmatiques ou rhétoriques, voire en sociolinguistique, de par sa fonction de gap filler, l'auteur propose pour sa part une approche plus originale : il s'agit de tracer son évolution diachronique à partir de différentes hypothèses, pour élucider les circonstances de l'avènement de sa fonction métadiscursive actuelle en tant que locution. Le sémantisme de verbe de perception marquant un lien du vu au visible fournit une première explication de son évolution vers l'expression de la causalité portée par la locution. L'auteur privilégie l'hypothèse d'une dérivation à partir d'une forme interrogative du verbe, et réfute in fine celles d'une proposition principale complémentée par une subordonnée (you see that...) et d'une subordonnée à valeur adverbiale (as you see), en s'appuyant sur la Théorie des Opérations Énonciatives et en considérant notamment les variations pragamatiques liées à la position du marqueur dans les propositions, suivant qu'elle est initiale, médiane ou finale.

Christopher Desurmont (p. 208) poursuit avec la locution adverbiale de modalisation Just about comme "expression de l'approximation dans les procès ». Etudiée dans le cadre de la TOE, cette composition est d'abord interprétée à partir de son étymologie. L'auteur la comprend comme rendant compte d'un positionnement par rapport à un domaine notionnel : la relation prédicative y est validée, mais le procès y est représenté avec plus ou moins d'altérité par rapport à un centre organisateur, sans dépasser « un seuil critique » (p. 211) qui ne permettrait plus l'identification dudit procès. L'article étudie les gradations de cette distance, et par là-même de cette altérité, paradoxale, mais au fondement de la valeur modale de la locution, en démontrant l'apport indéniable de l'approche topologique du domaine notionnel pour ce faire.

Pierre Cotte (p. 224) clôt l'ouvrage avec une étude sur la locution idiomatique grammaticale There is no + gérondif. Cette expression de l'impossible, à la fois figée et productive, tire sa valeur de sa construction, mais aussi de son contexte : l'auteur montre en effet comment sa subjectivité, « interne à la conscience » (p. 230) en dehors de tout sujet grammatical, témoigne de la motivation de l'émetteur : le préconstruit dont rend compte la subordonnée est ainsi annulé dans la principale, et c'est la portée 
de la négation qui scelle l'inexistence d'un procès désiré par le locuteur. Alors même que l'analyse de chacun des éléments de cette phrase complexe révèle leur fonctionnement tout à fait attendu en langue anglaise, c'est leur combinaison, la mise en locution, qui exprime alors un « impossible a posteriori » (p. 238). La démonstration très structurée et argumentée proposée ici ouvre des perspectives stimulantes à la fois quant à la conception même de locution et à la réflexion sur les procès.

11 On le voit, la recherche sur les locutions, dont l'étude est loin d'être épuisée, s'avère productive et variée. Ces expressions, originales à plus d'un titre - «Les équivalents d'une locution ou d'un proverbe ne les remplacent pas ", nous dit Berman [1999: 65] (emphase de l'auteur) -, peuvent prendre de multiples facettes qui ne sont bien sûr pas toutes abordées dans cette publication et font que cette collection d'articles ne suit pas toujours un fil conducteur explicite; pour autant, elle offre un renouvellement de perspective bienvenu en élargissant la compréhension d'un phénomène langagier vaste et multiforme et en mêlant les approches méthodologiques. Accessible et clairement présentée, elle intéressera en conséquence de nombreux chercheurs anglicistes, que ce soit en linguistique, pragmatique, sémantique, lexicologie, lexicographie ou traductologie, pour ne citer que quelques-uns des domaines concernés, car ce volume, de format fort pratique qui plus est, se prête tout autant à la consultation ponctuelle qu'à une lecture extensive.

\section{BIBLIOGRAPHY}

BERMAN Antoine, 1999, La traduction et la lettre ou l'auberge du lointain, Paris : Seuil.

BouRdiEu Pierre, 1982, Ce que parler veut dire, l'économie des échanges linguistiques, Paris :

Fayard.

DuboIs Marie-Marguerite, 1973, Dictionnaire de locutions français-anglais, Paris : Larousse.

MitTERAND Henri, 1976, Les mots français, Paris : PUF.

TOURNIER Jean, 2004, Précis de lexicologie anglaise, Paris : Ellipses.

\section{NOTES}

1. Tournier [2004: 15] distingue par exemple les lexies prépositionnelles des lexies complexes, telles que «les phrases lexicalisées, comme les proverbes ou certaines citations passées dans l'usage ».

2. On retrouve cette approche chez Marguerite-Marie Dubois [1973: v], qui signale «toute une gamme de complexes spontanés consistant à unir plusieurs constituants de façon rituelle [...] toujours utilisés sous forme de blocs ». 


\section{AUTHOR}

\section{FRÉDÉRIQUE BRISSET}

Frédérique Brisset, EA CECILLE, Université de Lille, France.

Spécialiste de traduction et tradictologie (anglais français), Frédérique Brisset est docteure en études anglophones de l'Université Sorbonne Nouvelle et maître de conférences honoraire. Ses recherches portent principalement sur la traduction audiovisuelle, la lexicologie contrastive, la stylistique et l'herméneutique de la traduction. 